\title{
Modified Gravity Model with Dark Energy for Accelerating Universe
}

\author{
R. K. Das* \\ Dept. of Physics, Charuchandra College, University of Calcutta, Kolkata, India \\ *Corresponding Author: rkdas_171171@rediffmail.com
}

Available online at: www.isroset.org

Received 20/Dec/2017, Revised 04/Jan/2018, Accepted 25/Jan/2018, Online 28/Feb/2018

\begin{abstract}
Dust dominated cosmological model has many problems to explain accelerating universe. Many models have been proposed which tells that dark energy causes acceleration of the universe. The evolution of the universe is described by Einstein's theory of general relativity. This paper examines the possibility of solving problem of accelerating universe by modifying gravity with generalised non-minimally coupled scalar field model. We have generalized the scalar-curvature coupling model $\xi \Phi^{2} R$ of Higgs inflation to $\xi \Phi^{a} R^{b}$. The field equations of a generalized $F(R, \phi)$ type gravity model, in which there is an arbitrary coupling between matter and geometry, are obtained. The equations of motion for test particles are derived from a variational principle and checked for energy conditions in order to explain acceleration of the universe. The developed dynamics has been studied under different energy conditions which has been implemented in graphs. The presence of scalar-gravity interaction term is not dominated over the first two terms of Klein-Gordon equation showing scalar-gravity interaction is not viable for accelerating universe.
\end{abstract}

Keywords-Universe, Dark Energy, Dark Matter, Modified Gravity, Accelarating Universe

\section{INTRODUCTION}

The discovery of the accelerating expansion of the universe is a milestone for cosmology. The evolution of the universe is described by Einstein's theory of general relativity. Many models of universe are proposed under the hypothesis that dark energy causes acceleration of the universe.

Recent astrophysical observations have provided the astonishing result that around $95-96 \%$ of the content of the Universe is in the form of dark matter and dark energy, with only about 4-5\% being represented by baryonic matter [1-4]. More intriguingly, around $70 \%$ of the energy-density is in the form of what is called "dark energy", and is responsible for the acceleration of the distant type Ia supernovae $[5,6]$. Hence, today's models of astrophysics and cosmology face two fundamental problems, the dark energy problem, and the dark matter problem, respectively. Although in recent years many different suggestions have been proposed to overcome these issues, a satisfactory answer has yet to be obtained.

In this paper we have generalized the scalar-curvature coupling model $\xi \Phi^{2} R$ of Higgs inflation to $\xi \Phi^{a} R^{b}$, then checked for this generalized scalar field theory $F(R, \phi)$, where the scalar inflation field is coupled to the Ricci scalar $\mathrm{R}$ through a non-minimal coupling $\xi$,to describe the accelerating universe.
This paper has been written into four distinct sectionsIntroduction, Theory and Calculations, Results and Discussion and Conclusion. Introduction section gives the brief review of literature related to our present work. Theory and calculation contains the theory related to our work and calculations done by us. Results and discussion shows the results of our work and related graphs obtained from our calculations. Conclusion part gives the major conclusion drawn from our results.

\section{THEORY AND CALCULATIONS}

\section{FIELD EQUATIONS IN $F(R, \phi)$ TYPE MODEL}

The universe has an accelerated expansion, but many current theories give an expansion that slows down due to gravity. So we need a pressure that works in the opposite way of gravity. This pressure is called the dark energy and should have no coupling with radiation. In the last decade numerous dark energy models were explored. These dark energy models can roughly be divided into three types: A true cosmological constant, physical dark energy models and geometrical dark energy models.

The cosmological constant $\left(w_{0}=-1\right)$ is still in good agreement with data, but other possibilities are still plausible. 
For example, it is possible to modify the stress-energy tensor replacing the cosmological constant with a scalar field $\varphi$ and many different models can be constructed using different Lagrangians and exploiting various mechanisms to produce the accelerated phase $[6,7]$.

In this section we will explain the idea by Bezrukov and Shaposhnikov and carry out the non-minimal coupling to a generalized and try to find out the possibility [8]. As mentioned in the previous section, a scalar field is an essential ingredient for inflationary models. The only known scalar particle in the Standard Model is the Higgs boson. The potential for the Higgs field is

$V(H)=\lambda\left(H^{\dagger} H\right)^{2}$

where $\mathrm{H}$ is the Higgs doublet

$H=\left(\begin{array}{l}\phi^{0} \\ \phi^{+}\end{array}\right)$

where $\phi^{0}$ and $\phi^{+}$are complex scalar fields. We can fix a gauge (the unitary gauge) in which

$H=\left(\begin{array}{c}\frac{\phi}{\sqrt{2}} \\ 0\end{array}\right)$

where $\phi$ is now a real scalar field with vacuum expectation value $\langle\phi\rangle=v$. The potential then becomes the familiar "Mexican hat" potential,

$V(\phi)=\frac{1}{4} \lambda\left(\phi^{2}-v^{2}\right)^{2}$

We now consider a chaotic inflationary scenario, where the Higgs field $\phi$ has a large initial value of $\mathrm{O}\left(M_{P}\right)$. Since $\mathrm{v}<<M_{P}$, we can safely neglect the Higgs vacuum expectation value $\mathrm{v}$ in the potential (equation 4).Thus, the Higgs potential during chaotic inflation is effectively a quartic potential with self-coupling $\lambda$. The value of $\lambda$ is not yet known, but we can calculate an allowed range. The Higgs mass is expected to lie in the range $(114-185) \mathrm{GeV}$. The lower bound is set by experiments, whereas the upper bound is needed for stability of the Standard Model all the way up to the Planck scale.

In this section we include a non-minimal coupling term in the Higgs action. This changes the potential of our theory and therefore the constraints on $\lambda$. Specifically the quartic self-coupling can be $\lambda \sim 10^{-1}$ if $\xi \sim-10^{4}$ [9]. This allows the Higgs boson to be the inflaton, which is a very nice feature of non-minimal inflation, because we can now explain inflation from the Standard Model. The total action we thus consider consist of the Standard Model action, the Einstein-Hilbert action and the non-minimal coupling term.

$S=\int d^{4} x \sqrt{-g}\left[\mathrm{~L}_{S M}+\frac{1}{2} M_{P}^{2} R-\xi H^{\dagger} H R\right]$

where $\mathrm{L}_{M}$ is the Standard Model Lagrangian. If we again take the unitary gauge for the Higgs doublet $\mathrm{H}$ and neglect all the gauge and fermion interactions for now, we find the action for the Higgs boson

$S=\int d^{4} x \sqrt{-g}\left[\frac{1}{2}\left(M_{P}^{2}-\xi \phi^{2}\right) R-\frac{1}{2} g^{\mu v} \partial_{\mu} \phi \partial_{v} \phi-\frac{\lambda}{4}\left(\phi^{2}-v^{2}\right)^{2}\right]$

where again the Higgs VEV appears with a value $v=246$ $\mathrm{GeV}$. This is the action in the so-called Jordan frame. We can now get rid of the non-minimal coupling of $\phi$ to gravity by making a conformal transformation to the Einstein frame. The reason that we do this is to calculate the power spectrum , which gives us constraints on the inflate on potential. The power spectrum was derived for a minimally coupled inflaton field, and therefore we perform a conformal transformation aiming to remove the non-minimal coupling term from the action. To do the conformal transformation we can write our action

as,

$S=\int d^{4} x \sqrt{-g}\left[\frac{1}{2} M_{P}^{2} \Omega^{2} R-\frac{1}{2} g^{\mu v} \partial_{\mu} \phi \partial_{\nu} \phi-\frac{\lambda}{4}\left(\phi^{2}-v^{2}\right)^{2}\right]$

Where,

$\Omega^{2}=\frac{M_{P}^{2}-\xi \phi^{2}}{M_{P}^{2}}$

We have taken a generalize situation by choosing the action for generalized $\mathrm{f}(\phi, \mathrm{R})$ gravity [10]. So we have

$s=\int d^{4} x \sqrt{-g}\left[\frac{f(\phi, R)}{2 \kappa^{2}}+\frac{1}{2} g^{\mu v} \partial_{\mu} \phi \partial_{\nu} \phi+V(\phi)\right]_{(9)}$

We take,

$\frac{f(\phi, R)}{\kappa^{2}}=\frac{R}{\kappa^{2}}+\frac{\xi \phi^{i} R^{B}}{M^{i+2 B-4}}$ 
$V(\phi)=\frac{\lambda \phi^{4}}{4}$

$\kappa^{2}=1 / M_{p}^{2}$

$\xi$ as a dimensionless coupling constant .

Now varying the action with respect to $\left(g^{\mu v}\right)$ we obtain field equationslcite $\{$ Chakravarty:2013eqa $\}$,

$F R_{\mu \nu}-\frac{1}{2} f g_{\mu \nu}-\nabla_{\mu} \nabla_{v} F+g_{\mu \nu} \square F$

$=\kappa^{2}\left(\nabla_{\mu} \phi \nabla_{\nu} \phi-\frac{1}{2} g_{\mu \nu} \nabla^{\rho} \phi \nabla_{\rho} \phi-V g_{\mu \nu}\right)$

$F=\frac{\partial f}{\partial R}=1+\left(\frac{\xi B R^{-1+B}}{M^{i+2 B-2}}\right) \phi^{i}$

$G_{\mu \nu}=\frac{1}{f_{R}}\left[\left(\nabla_{\mu} \nabla_{v}-g_{\mu v} \square\right) f_{R}+\frac{1}{2} g_{\mu \nu}\left(f-f_{R} R\right)+\right.$

$\left.\kappa^{2}\left(\nabla_{\mu} \phi \nabla_{\nu} \phi-\frac{1}{2} g_{\mu \nu} \nabla^{\rho} \phi \nabla_{\rho} \phi-V g_{\mu \nu}\right)\right]$

$T_{\mu v}=\frac{1}{f_{R}}\left[\frac{1}{\kappa^{2}}\left(\nabla_{\mu} \nabla_{v}-g_{\mu v} \square\right) f_{R}+\right.$

$\left.\frac{1}{2} g_{\mu \nu}\left(f-f_{R} R\right)+\left(\nabla_{\mu} \phi \nabla_{\nu} \phi-\frac{1}{2} g_{\mu \nu} \nabla^{\rho} \phi \nabla_{\rho} \phi-V g_{\mu \nu}\right)\right]$

taking 00 component of equation (16)

$T_{00}=\frac{1}{f_{R}}\left[\begin{array}{l}\frac{1}{\kappa^{2}}\left\{f_{\mathrm{RR}}(-3 H) \dot{R}+\frac{1}{2}\left(f-f_{R} R\right)\right\}+ \\ \left(\frac{1}{2} \dot{\phi}^{2}-v(\phi)\right)\end{array}\right]$

taking space part of equation (16)

$T_{i i}=\frac{a^{2}}{f_{R}}\left[\frac{1}{\kappa^{2}} f_{R R R} \dot{R}^{2}+f_{R R}(\ddot{R}+R H \dot{R})\right.$

$\left.-\frac{1}{2}\left(f-f_{R} R\right)-\left(\frac{1}{2} \dot{\phi}^{2}-v(\phi)\right)\right]$
Scalar field $\phi=\phi$ (t) reduces to the form to get the solution for $\phi$ we need to solve the equation (13)

$\ddot{\phi}+3 H \dot{\phi}-V_{, \phi}+\frac{f_{, \phi}}{2 \kappa^{2}}=0$

$\ddot{\phi}+3 H \dot{\phi}+\lambda \phi^{3}+\frac{\kappa^{2} \xi R^{B}}{M^{i+2 B-4}} i \phi^{i-1}=0$

For late time acceleration we assume that there exists an exact power law solution to the modified field equations

$a(t)=a_{0} t^{m}$

where, $\mathrm{m}$ is a positive real number . if $0<m<1$, then the required power law solution is decelerating while for $m>1$ it exhibits accelerating behavior. Ricci scalar part for large time tends to very small and the value of $m$ being very large ,the last term of equation (20) can be treated as the perturbation part of the non-linear equation (20) as,

$a \propto t^{m}$

$R=-6\left(\dot{H}+2 H^{2}\right)$

$R=-\frac{6 m}{t^{2}}(-1+2 m)$

$R \propto \frac{1}{t^{2}}$

as $\mathrm{t}$ is large the, $\mathrm{R}->0$

$H=\frac{m}{t}$, for large time the second term of equation (20) also can be treated as perturbation part. Hence the equation becomes,

$\ddot{\phi}-\lambda \phi^{3}=0$

So,

$\phi=-\sqrt{\frac{2}{\lambda}} \frac{1}{t}$ 


\section{RESULTS AND DISCUSSION}

\section{ENERGY CONDITIONS CALCULATION}

The Einstein field equation $G_{\mu \nu}=8 \pi T_{\mu \nu}$ describes the relationship between the stress-energy tensor $T_{\mu \nu}$ of matterfields and the geometrical properties of space-time. In principle, one can take any metric $g_{\mu \nu}$ as long as its second partial derivatives exist, the stress-energy tensor corresponding to that metric can be produced. In this way, exact solutions to the equation can easily be constructed, but the stress-energy tensors will not necessarily be physically reasonable. It is therefore useful to impose one or more energy conditions. Energy conditions serve to precisely codify certain ideas about what is physically reasonable. We will study four energy conditions that are in standard usage. These are: the weak energy condition (WEC), the null energy condition (NEC), the dominant energy condition (DEC), and the strong energy condition (SEC) [10].

After calculating NEC WEC DEC in convenient way and plotting we get,

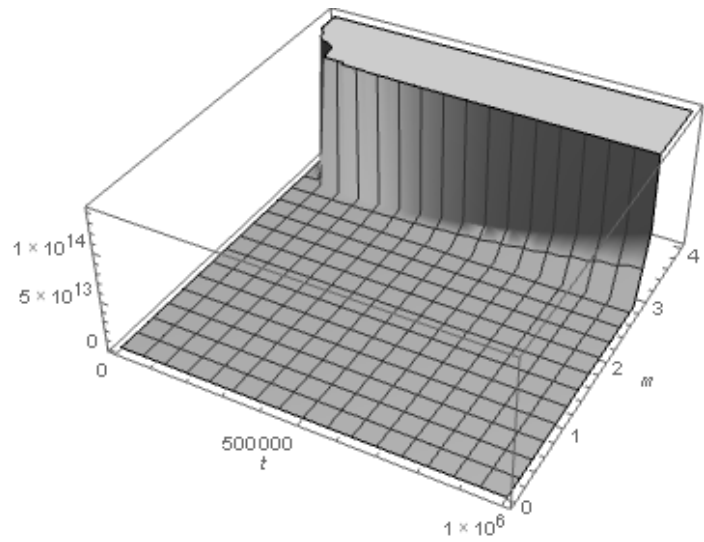

DEC taking values $\mathrm{B}=1, \mathrm{a} 0=7, \xi=1, \mathrm{i}=3, \kappa=1, \mathrm{M}=1$ ,$\lambda=10^{-1}$

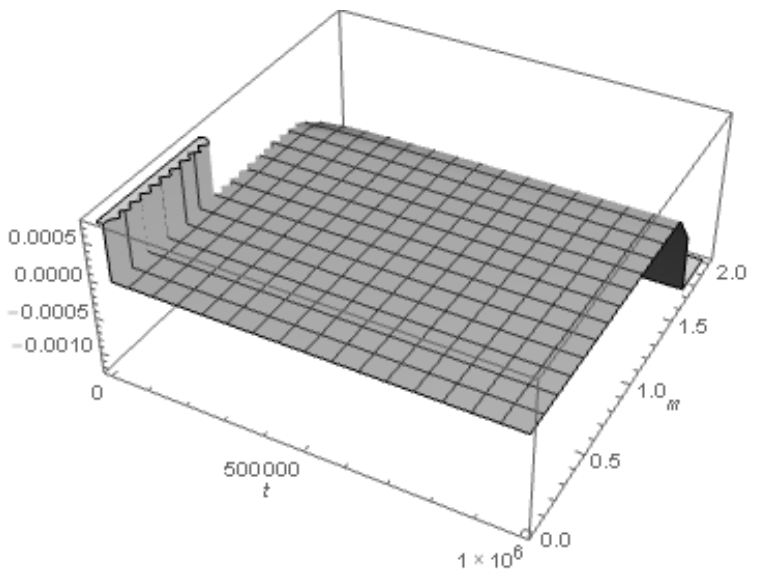

NEC taking values $\mathrm{B}=1, \mathrm{a} 0=7, \xi=1, \mathrm{i}=3, \kappa=1, \mathrm{M}=1$ ,$\lambda=10^{-1}$

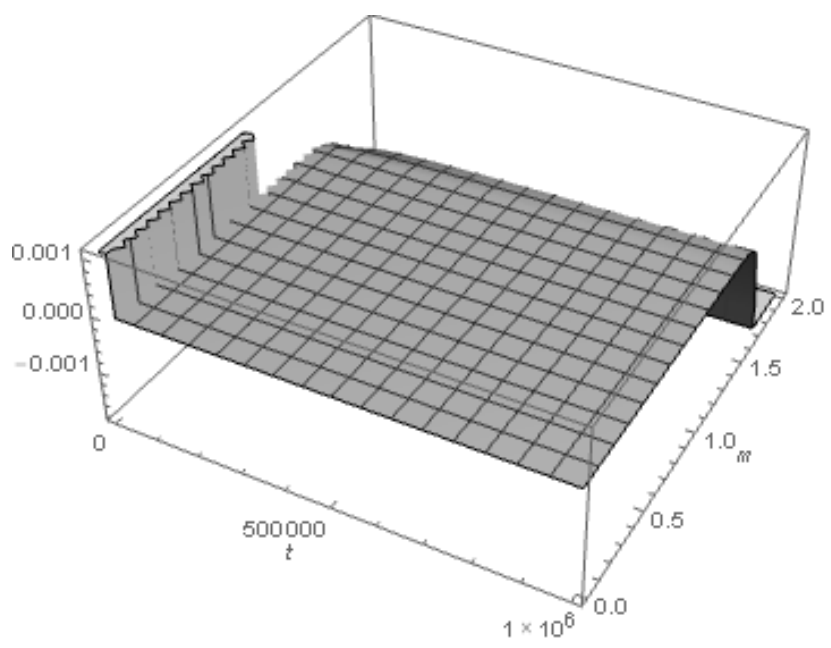

SEC taking values $\mathrm{B}=1, \mathrm{a} 0=7, \xi=1, \mathrm{i}=3, \kappa=1, \mathrm{M}=1$ ,$\lambda=10^{-1}$

We found SEC is being violated as it does for an accelerating universe so we checked the cases of NEC and DEC .We see violation of NEC here too and as a consequence WEC cannot be satisfied, which we were not expecting to be violated.

This may be consequence of the over simplification of the $\varphi$ solution so as a future work we head to solve the equation more rigorously.

As for inflationary cosmology, there are specific slow-roll conditions that can assess whether cosmic acceleration arises at late time.In the usual case of a single field, the relevant quantities to compute are,

$\grave{\mathrm{o}}=\frac{M^{2}}{2}\left(\frac{V_{, \phi}}{V}\right)^{2}$

and,

$\eta=\frac{M^{2} V, \phi \phi}{V}$

In our case, the condition ò $<<1$ cannot be hold with the potential equation (20), since

ò $\propto \frac{M^{2} \phi^{2}}{V}$

is a divergent quantity when the higgs field settles in the minimum and the potential vanishes. 
In fact the presence of scalar-gravity interaction term $f(\phi, R)$ is not dominated over the first two terms of KleinGordon equation (4),showing scalar-gravity interaction is not a viable for accelerating universe .

\section{CONCLUSIONS}

In this paper we have examined the possibility of solving problem of accelerating universe by modifying gravity with generalised non-minimally coupled scalar field model. We have generalized the scalar-curvature coupling model $\xi \Phi^{2} R$ of Higgs inflation to $\xi \Phi^{a} R^{b}$. The field equations of a generalized $F(R, \phi)$ type gravity model, in which there is an arbitrary coupling between matter and geometry, are obtained. The equations of motion for test particles are derived from a variational principle and checked for energy conditions in order to explain acceleration of the universe. The developed dynamics has been studied under different energy conditions which has been implemented in graphs.

\section{ACKNOWLEDGMENT}

I am thankful to Principal and Department of Physics, Charuchandra College, University of Calcutta for giving me support.

\section{REFERENCES}

[1] A. G. Riess, A.V. Filippenko, P. Challos, A. Clocchiattia, A. Diercks, "Observational evidence from Supernovae for an accelerating universe and a cosmological constant", The Astronomical Journal, vol.116, pp. 10091038,1998

[2] S. Perlmutter ,G. Aldering, G. Goldhaber, R.A. Knop, P. Nugent, "Measurements of Omega and Lambda from 42 high-Redshift Supernovae”, Astrophysical Journal, vol. 517, pp. 565-586, 1999.

[3] P. de Bernardis, P.A.R. Ade, J.J. Bock, J.R. Bond, J. Borill, "A flat universe from high resolution maps of the Cosmic Microwave background radiation", Nature , vol.404, pp.955-959, 2000.

[4] S. Hanany , P. Ade, A. Balbi, J. Bock, J. Borrill, "Maxima-I: A measurement of cosmic microwave background Anisotropy on angular scales of 10.5 ", Astrophysical Journal, vol545, L5-L9, 2000.

[5] P. J. E. Peebles, B. Ratra "The cosmological constant and dark energy", Reviews of Modern Physics, vol.75, pp. 559,2003

[6] T. Padmanabhan, “ Cosmological constant- the weight of the vacuum”, Physics Reports, vol.380, pp. 235-320, 2003
[7] Edmund, J. Copeland, M. Sami, and Shinji Tsujikawa. "Dynamics of dark energy.", International Journal of Modern Physics D, vol.15, pp. 1753-1936, 2006

[8] Fedor, L.Bezrukov and Mikhail Shaposhnikov, "The Standard Model Higgs boson as the inflaton.", Physics Letters B, vol.659, pp.703-706, 2008

[9] Girish Chakravarty, Subhendra Mohanty, and Naveen K. Singh, "Higgs Inflation in $F(R, \phi)$ Theory.", International Journal of Modern Physics D , vol.23, Issue4, pp. 1450029-43,2014

[10] Stephen W. Hawking and G. F. R. Ellis, The Large Scale Structure of Space-Time, (Cambridge Monographs on Mathematical Physics). Cambridge University Press, 1975. ISBN 0521099064. 